\title{
The Mid-term outcomes of ankle Dias-Tachdjian PEER physeal fracture in children treat by ORIF with One- stage medial-lateral combined incision
}

\section{Feng XIANG}

Hunan Provincial People's Hospital

\section{Bo LI}

Hunan Provincial People's Hospital

\section{Sheng XIAO}

Hunan Provincial People's Hospital

JieWen ( $\nabla$ cashwj@qq.com )

Hunan Provincial People's Hospital

\section{Hong LIU}

Hunan Provincial People's Hospital

Xin LI

Hunan Provincial People's Hospital

\section{Ke FANG}

Hunan Provincial People's Hospital

\section{Zhongwen TANG}

Hunan Provincial People's Hospital

\section{Shu CAO}

Hunan Provincial People's Hospital

\section{Bo Lee}

Hunan Provincial People's Hospital

\section{Fanling LI}

Hunan Provincial People's Hospital

\section{Kewei ZHU}

Hunan Provincial People's Hospital

\section{Research article}

Keywords: Dias-Tachdjian, physeal fracture, medial-lateral combined incision, Mid-term outcomes

Posted Date: March 27th, 2020

DOI: https://doi.org/10.21203/rs.3.rs-18892/v1 
License: (9) (1) This work is licensed under a Creative Commons Attribution 4.0 International License. Read Full License 


\section{Abstract}

Objectives: To report the mid-term outcomes and complications of ankle Dias-Tachdjian pronation-eversion external rotation (PEER) physeal fracture in children treat by ORIF with combined medial and lateral incision.

Materials and Methods: A total of 21 children (12 male and 9 female patients with ankle Dias-Tachdjian PEER physeal fracture, underwent open reduction treatment with combined medial and lateral incision between Jan, 2015 and Oct, 2017. The surgical outcomes were assessed by X-ray film after operation. The lateral distal tibia angle (LDTA) was measured to evaluate angular deformity and the $X$ rays was taken to evaluate the premature physeal arrest.

Result: All the patients were followed up for an average time of 20.1 months (ranging from 17 to 25 months). Bone healing was achieved in all cases. American Orthopedic Foot and Ankle Society-Ankle and Hindfoot score(AOFAS-AH) of the patients improved from median $31(11,38)$ before operation to median $68(63,73)$ postoperation, median score $91(87,96)$ at last follow-up. Lateral distal tibia angle (LDTA) of the patients improved from $70.5 \pm 4.9$ before operation to $90.0 \pm 1.2$ post-operation, measure $90.6 \pm 3.7$ at last follow-up. The differences between pre and post operation and the differences between pre-operation and last follow-up in both findings were statistically significant $(P<0.05)$. There is no premature physeal closure, ankle deformity in 19 cases. They could normally exercise and take part in normal sport activities. The remaining 2 cases had physeal bone bridge and premature physeal closure but could still carry on daily activities and thus were categorized as good.

Conclusion: ORIF with one-stage medial-lateral combined incision for ankle Dias-Tachdjian PEER physeal fracture in children can decrease the rate of premature physeal closure and has excellent efficacy for fracture union and the recovery of ankle functions.

\section{Introduction}

The physeal fracture is a common type of fractures, accounting for $20 \%$ to $30 \%$ of all fractures in children. The distal tibial type constitutes $1 / 3$ of physeal fractures [1] and is primarily induced by falling injuries [2]. The most important complication is premature physeal closure-related limb deformity. Incomplete reduction and multiple closed reductions can increase the risk of premature physeal closure. Around $25 \%$ of distal tibial physeal fractures are accompanied by fibular fracture. The stable fibula after reduction is beneficial for healing of distal tibial physeal injury and can shorten the healing time of tibial physis [3]. The current treatment strategies include expectant treatment, fixation with external fixator, internal fixation with locked reconstruction plate in combination with Kirschner wire, and fixation with absorbable screw [4,5].

In the present study, we discussed the mid-term outcomes and complications of combined medial and lateral incision for the treatment of ankle Dias-Tachdjian PEER physeal fracture in children. Fracture healing and complications were also assessed via the imaging.

\section{Materials And Methods}

Study population 
Children with ankle fracture treated at our department between January 2015 and October 2017 were enrolled in the current study. All subjects met the following criteria: 1) ankle Dias-Tachdjian PEER physeal fracture diagnosed by two independent attending physicians, associate chief, or chief physicians reading the film; 2) fracture gap $>3 \mathrm{~mm}$ under Fluroscopy perspective after reductions once or twice under anesthesia and muscle relaxation; and 3) aged<15 years old. A total of 21 patients, 12 males and 9 females with an average age of $10.3 \pm 1.8$ years old ( 6 to 14 years old) were eligible. There were 13 cases of the right foot injury and 8 of the left foot injury; 12 cases were caused by falling injury and 9 cases by road traffic injury.

\section{Surgical procedures}

Placed in the supine position, the patient was given general anesthesia and the fracture end was fixed under the Fluroscopy. Lateral and medial longitudinal incisions were successively made and the skin, subcutaneous tissue and deep fascia were cut to fully expose the fracture ends. Fibular fracture was fixed with the locked reconstruction plate, which was proved rigid under the fluroscopy and direct vision. An incision was made in the medial tibia to remove the curled periosteum in the fracture (Figure 1).. After anatomical reduction of the distal tibia physis, the fracture was crossly fixed with the Kirschner wire $(1.6 \mathrm{~mm})$, which was also rigid under the Fluroscopy. Finally, the wound was rinsed, sutured and dressed.

\section{Data collection}

Lateral distal tibia angle (LDTA) in the anterior-posterior radiography pre- and post- operation and during the last-follow up were used as the evaluating indicators, which were measured as the angle of axial line of the tibia with tibia-ankle joint line. American Orthopedic Foot and Ankle Society-Ankle and Hindfoot (AOFAS-AH) were recorded before operation, post operation and the last follow up (Table 1)..

\section{Statistical analysis}

SPSS was used for statistical analysis. The AOFAS-AH score were expressed as the median(Min, Max). The LDTA degree were expressed as the mean \pm standard deviation (SD). All measured data during pre and post operation and the last-follow up were compared using the wilcoxon signed rank test. $\mathrm{P}<0.05$ was considered statistically different.

\section{Results}

All patients were followed up post operation, with the average follow-up period of $20.1 \pm 2.1$ months. Bone union was achieved in all 21 cases and no disunion was seen. AOFAS-AH of ankle function where above 90 stood for excellent, 75-89 for good, 50-74 for fair, and below 50 for poor [5], were used to evaluate the pain, walking, active movement, ankle joint function, stability and deformity. American Orthopedic Foot and Ankle Society-Ankle and Hindfoot score(AOFAS-AH) of the patients improved median $31(11,38)$ before operation to median 68(63,73) post-operation, median score $91(87,96)$ at last follow-up. There was a significant difference among the preoperation, postoperative and last follow-up scores $(P<.05)$. A total of 16 cases were categorized 
as excellent and 5 cases as good. During the last follow up, 19 patients reported normal ankle joint ability and no discomfort while other two patients reported slightly limited activity of ankle joint. Lateral distal tibia angle (LDTA) of the patients improved from $70.5 \pm 4.9$ before operation to $90.0 \pm 1.2$ post-operation, measure $90.6 \pm 3.7$ at last follow-up. The differences between pre and post operation and the differences between pre-operation and last follow-up were statistically significant $(\mathrm{P}<.05)$. During the last follow-up, 19 cases had normal Lateral distal tibia angle (LDTA) and no ankle varus whereas two patients, a 13-year old girl and an 11-year old boy, experienced slight ankle varus that did not affect their daily activities. The girl's physeal plate was almost mature and the varus deformity did not worsen. The boy had the growth potential, and the ankle varus progressively aggravated leading to the second surgery, which eventually produced satisfactory effect (Table 1).. Among 21 cases, 2 had pressure ulcer in the heel 7 weeks after removing gypsum, which healed following the change of dressing and antibiotics. Typical cases are shown in Figure 2, 3 and 4.

\section{Discussion}

The distal tibial physeal fracture is very common in childhood, making up for around $1 / 3$ of all fractures in children [1]. Salter-Harris classification is most widely used for physeal fractures; however, it is limited to ankle fracture. In 1978, Dlas and Tachdjian [8] modified Lauge-Hansen classification and grouped the child ankle fractures into four subtypes comprising the Salter-Harris classification. PEER fracture is the typical distal tibial Lauge-Hansen I or II fracture complicated with fibular fracture. In the current study, all 21 patients had SalterHarris II fracture.

Stable distal tibial Salter-Harris II fracture without displacement usually requires the following treatment: short leg or bent long leg plaster fixation for 3-4 weeks followed by plaster cast or walking splint for 2-3 weeks. For unstable displaced Salter-Harris I or II distal tibial phyeal fracture, acceptable or suitable reduction are still controversial. Barmada et al [7] made expectant treatment for distal tibial Salter-Harris I and II fractures finding the premature physeal closure in $60 \%$ of patients $(14 / 20)$ with fracture gap $>3 \mathrm{~mm}$ after reduction and $17 \%$ of patients (4/24) with fracture gap $<3 \mathrm{~mm}$. Yet, Park et al [8] found no significantly different effects of operative and nonoperative treatment for Salter-Harris II distal tibial fractures with residual gap of $>3 \mathrm{~mm}$. Nevertheless, the authors still considered that surgery might reduce the rate of premature physeal closure for PEER fracture for the high rate of premature physeal closure in this type of fracture, although surgery might not be very effective for patients with residual gap>3mm after reduction [9]. In the present study, we enrolled PEER patients, and conducted open reduction to reduce the rate of premature physeal closure, regardless of residual gap $>3 \mathrm{~mm}$. From the medial incision in the tibia, periosteum entanglement was observed in all 21 cases. The periosteum was pulled out and the second reduction was conducted, followed by Kirschner wire insertion to fix the fracture end, and maximally reduce the rate of premature physeal closure. Our results suggest that this type of fracture should be treated by open reduction and periosteum removal to maximally reduce the rate of premature physeal closure.

Fibular PEER fracture has not yet been fully elucidated. Blackburn et al [10] suggested that stable fibular fracture does not require internal fixation, while unstable fibula after reduction just needs closed reduction and internal fixation with Kirschner wire. In the static model of lower limb, Lambert and colleagues [11] found that fibula bore $1 / 6$ of body weight when the ankle joint stood. Strauss et al [12] proved that fibular fixation was helpful for maintenance of tibial reduction. However, Taylor and his team [13] reported that tibial fracture could 
heal even if fibula was not fixed and fibular fixation had no effect on postoperative tibial line of force. Furthermore, Weber et al [14] reported that fibular fixation could significantly reduce the movement of tibial fracture end for combined tibial and fibular fracture which was not strongly fixed but had no significant advantages for plate or bone nail fixation. In the present study, we use Kirschner wires to fix tibia and we used locked reconstruction plate to fix the fibula, result shows that our fixation partly reducing the residual gap in the physeal fracture of distal tibia and increasing the stability of fracture. The result demonstrates that both AOFAS-AH score and LDTA improved significantly from pre-operation to post-operation even the patient wearing a cast $(P<0.05)$, the AOFAS-AH score improved from post-operation to last follow-up $(P<0.05)$. The average LDTA maintain improved in last follow-up which indicate that the reduction and fixation bring good mid-term result. In addition, patients could do load-bearing and functional exercises very early after operation. They also benefited from fracture healing and reduction of complications.

One of the early and severe complications of ankle Dias-Tachdjian PEER physeal fracture is osteofascial compartment syndrome. To timely detect and prevent this complication, all patients were asked to raise the diseased limbs in order to observe levels of pain and toe activity. If this complication was suspected, plaster open arthrolysis was conducted.

One of the long-term complications is premature physeal closure, which occurs in up to $50 \%$ of PEER fractures as reported by Michael et al [15]. However, in the present study, only two patients (9.5\%) had premature physeal closure in the $X$ rays, while the ankle point angle was within the normal range, and there were no ankle varus and valgus deformity during the last follow up. The obvious inconsistencies with previous studies might be explained by small sample size and short follow-up period. Physeal arrest may appear 2 years post operation. Besides, medial-lateral incision in all cases, regardless of residual gap $>3 \mathrm{~mm}$ after reduction, fixes fibula and removes curled periosteum, thus reducing the incidence of premature physeal closure.

In conclusion, medial-lateral combined incision can decrease the incidence of premature physeal closure regardless of residual gap $>3 \mathrm{~mm}$ after reduction and thus should be a treatment choice for PEER fracture.

\section{Declarations}

Conflicts of Interest and Source of Funding: The authors declare that they have no competing interests. There was no funding support for this study.

Ethics approval: This study was approved by the ethic committee of Hunan Provincial People's Hospital, the First Affiliated Hospital of Hunan Normal University. All procedures performed in studies involving human participants were in accordance with the ethical standards of the institutional research committee and with the 1964 Helsinki declaration and its later amendments or comparable ethical standards. Informed consent was waived by the committee because of the retrospective nature of the study. Informed consent was waived by the committee because of the retrospective nature of the study.

Authors' contributions: XF and BL contribute equally to this study, they share co-first author, JW conceived and coordinated the study, designed, FX performed and analyzed the experiments, BL wrote the paper. SX, HL, XL, $\mathrm{KF}$ and KZ did the follow-up with the patients. ZT and SC did the data analysis; FL carried out the data 
collection and revised the paper. All authors reviewed the results and approved the final version of the manuscript.

Acknowledgements: Not applicable.

\section{References}

[1] D'Angelo, F. Solarino, G. Tanas, D. et al.(2017) Outcome of distal tibia physeal fractures: a review of cases as related to risk factors.[J].Injury. 48 Suppl 3:S7-S11

[2] Kling, T. F., Bright, R. W., Hensinger, R.N (1984) Distal tibial physeal fractures in children that may require open reduction.[J].J Bone Joint Surg Am. 66(5):647-57

[3] Seel, E. H., Noble, S, Clarke, N. M. et al. (2011) Outcome of distal tibial physeal injuries.[J].J Pediatr Orthop B. 20(4):242-8

[4] Schurz, M., Binder, H., Platzer, P. et al.(2010) Physeal injuries of the distal tibia: long-term results in 376 patients.[J].Int Orthop. 4(4):547-52

[5] Podeszwa, D. A., Wilson, P. L., Holland, A. R. et al.(2008) Comparison of bioabsorbable versus metallic implant fixation for physeal and epiphyseal fractures of the distal tibia.[J].J Pediatr Orthop. 28(8):859-63

[6] Dias, L. S., Tachdjian, M. O. (1978) Physeal injuries of the ankle in children: classification.[J].Clin Orthop Relat Res. 136:230-3

[7] Barmada, A., Gaynor, T., Mubarak, S.J (2003) Premature physeal closure following distal tibia physeal fractures: a new radiographic predictor. J Pediatr Orthop. 23(6):733-739.

[8] Park, H., Lee, D. H., Han, S. H., et al. (2018) What is the best treatment for displaced Salter-Harris II physeal fractures of the distal tibia?[J].Acta Orthop. 89(1):108-112.

[9] Russo, F., Moor, M. A., Mubarak, S. J., Pennock, A. T. (2013) Salter-Harris Il fractures of the distal tibia: Soes surgical management reduce the risk of premature physeal closure? J Pediatr Orthop.33(5): 524-529.

[10] Blackburn, E. W., Aronsso, D. D., Rubright, J. H., et al. (2012) Ankle fractures in children.[J].J Bone Joint Surg Am. 94(13):1234-1244.

[11] Lambert, K. L. (1971) The weight -bearing function of the fibula. A strain gauge study[J]. J Bone Joint Surg Am, 53(3):507-513.

[12] Strauss, E. J., Alfonso, D, Kummer, F. J., et al. (2007) The effect of concurrent fibular fracture on the fixation of distal tibia fractures:a laboratory comparison of intramedullary nails with locked plates [J]. J Orthop Trauma, 21(3):172-177.

[13]Taylor, B.C., Hartley, B. R., Formaini, N, et al. (2015) Necessity for fibular fixation associated with distal tibia fractures [J]. Injury. 46(12):2438-2442. 
[14] Weber, T. G., Harrington, R. M., Henley, M. B., et al.(1997) The role of fibular fixation in combined fractures

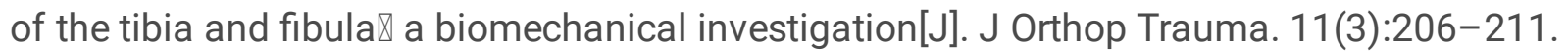

[15] Karlikowski, M., Sulko, J. (2018)Physeal fractures of the lower leg in children and adolescents: Therapeutic results, pitfalls and suggested management protocol - based on the experience of the authors and contemporary literature.[J].Adv Med Sci. 63(1):107-111

\section{Table}

Table 1 General information of selected children

\begin{tabular}{|c|c|c|c|c|c|c|c|c|c|c|}
\hline \multirow[t]{2}{*}{ No } & \multirow[t]{2}{*}{ Gender } & \multirow[t]{2}{*}{ Age/yo } & \multirow[t]{2}{*}{ Left/Right } & \multirow{2}{*}{$\begin{array}{l}\text { FU } \\
\text { Time/mo }\end{array}$} & \multicolumn{3}{|l|}{ AOFAS } & \multicolumn{3}{|l|}{ LDTA } \\
\hline & & & & & PreOP & PostOP & $\begin{array}{l}\text { Last } \\
\text { FU }\end{array}$ & PreOP & PostOP & $\begin{array}{l}\text { Last } \\
\text { FU }\end{array}$ \\
\hline 1 & $M$ & 8 & $\mathrm{R}$ & 19 & 31 & 63 & 91 & 68 & 88 & 90 \\
\hline 2 & $\mathrm{~F}$ & 10 & L & 21 & 33 & 73 & 95 & 70 & 90 & 92 \\
\hline 3 & $\mathrm{~F}$ & 11 & $\mathrm{R}$ & 18 & 34 & 66 & 87 & 65 & 87 & 105 \\
\hline 4 & $M$ & 6 & $\mathrm{R}$ & 20 & 11 & 65 & 96 & 60 & 89 & 91 \\
\hline 5 & $M$ & 9 & $\mathrm{R}$ & 22 & 31 & 66 & 91 & 64 & 90 & 90 \\
\hline 6 & $M$ & 8 & L & 24 & 28 & 71 & 92 & 70 & 91 & 89 \\
\hline 7 & $\mathrm{~F}$ & 11 & $\mathrm{R}$ & 17 & 34 & 68 & 89 & 72 & 89 & 91 \\
\hline 8 & $M$ & 10 & L & 20 & 31 & 68 & 96 & 75 & 90 & 88 \\
\hline 9 & $M$ & 14 & $\mathrm{R}$ & 19 & 38 & 71 & 91 & 70 & 92 & 89 \\
\hline 10 & $\mathrm{~F}$ & 9 & $\mathrm{R}$ & 21 & 27 & 68 & 91 & 80 & 90 & 91 \\
\hline 11 & $M$ & 10 & L & 18 & 31 & 73 & 96 & 75 & 90 & 89 \\
\hline 12 & $M$ & 11 & $\mathrm{R}$ & 25 & 33 & 71 & 92 & 70 & 91 & 90 \\
\hline 13 & $\mathrm{~F}$ & 13 & L & 20 & 34 & 73 & 88 & 76 & 90 & 88 \\
\hline 14 & $\mathrm{~F}$ & 10 & L & 18 & 28 & 68 & 91 & 78 & 88 & 87 \\
\hline 15 & $M$ & 11 & $\mathrm{R}$ & 19 & 31 & 63 & 89 & 70 & 90 & 90 \\
\hline 16 & $\mathrm{~F}$ & 13 & $\mathrm{R}$ & 22 & 34 & 73 & 92 & 72 & 88 & 95 \\
\hline 17 & $M$ & 9 & L & 21 & 27 & 63 & 91 & 68 & 90 & 91 \\
\hline 18 & $M$ & 10 & $\mathrm{R}$ & 18 & 31 & 73 & 95 & 69 & 91 & 90 \\
\hline 19 & $F$ & 11 & L & 19 & 33 & 71 & 92 & 70 & 90 & 90 \\
\hline 20 & $M$ & 12 & $\mathrm{R}$ & 20 & 31 & 68 & 89 & 74 & 89 & 88 \\
\hline 21 & $\mathrm{~F}$ & 10 & $\mathrm{R}$ & 22 & 34 & 65 & 92 & 64 & 90 & 89 \\
\hline
\end{tabular}




\section{Figures}
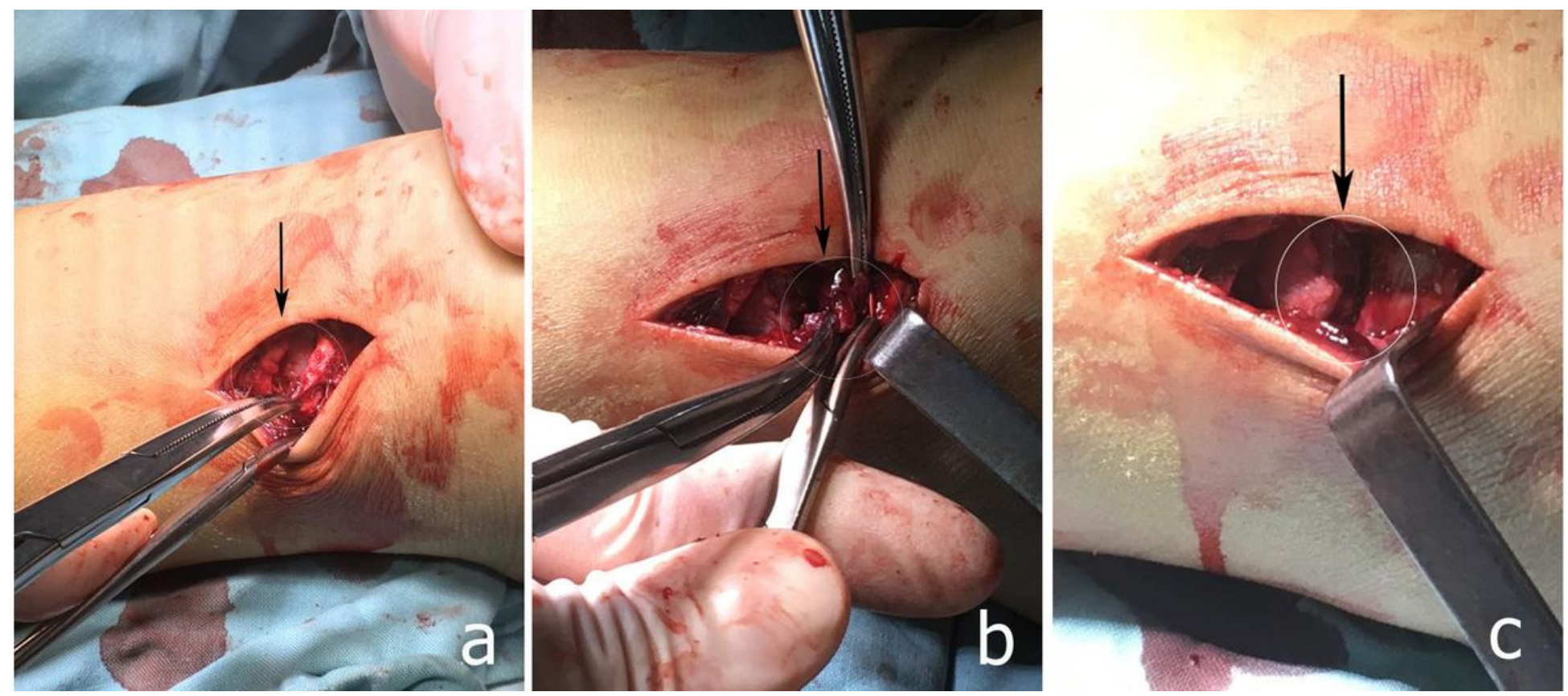

\section{Figure 1}

Intra-operative photographs. (a) Periosteum embeded in the fracture end, blocking the reduction; (b) periosteum was removed from the fracture end; (c) fracture end after removing periosteum.

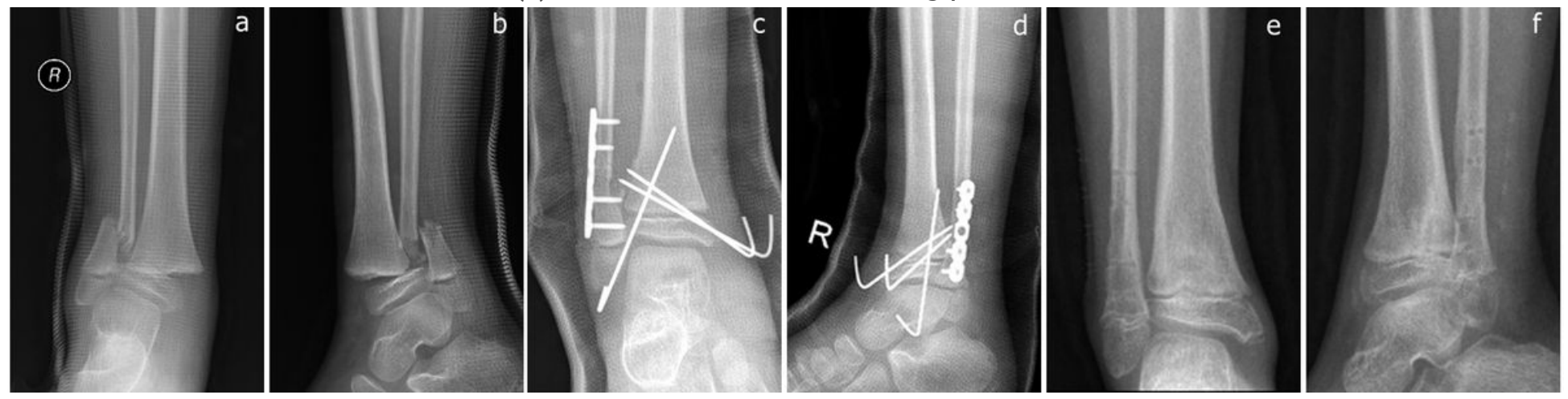

\section{Figure 2}

Boy, age 6, Case No.4, diagnosed the Dias-Tachdjian PEER physeal fracture of right distal tibial epiphysis and fibula, AOFAS-AH scoring 11 during preoperative assessment, 65 postoperation and 96 at 20 months follow-up. LDTA was 60 degree in the preoperative assessment, 89 degree postoperation and 91 degree at 20 months follow-up. 

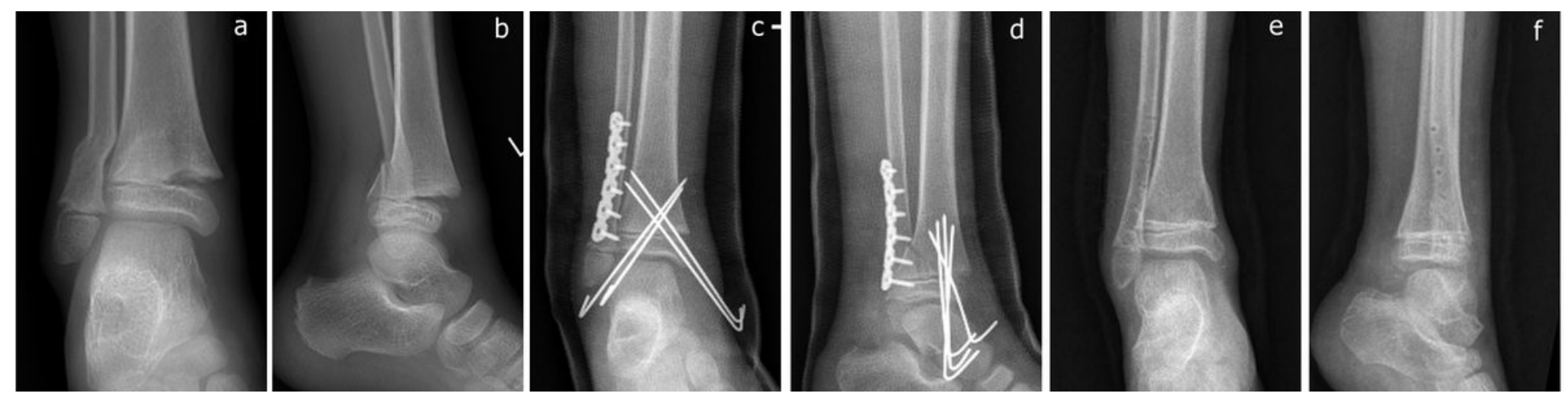

\section{Figure 3}

Girl, age 10, Case No.14, diagnosed the Dias-Tachdjian PEER physeal fracture of left distal tibial epiphysis and fibula, AOFAS-AH scoring 28 during preoperative assessment, 68 postoperation and 91 at 18 months follow-up. LDTA was 78 degree in the preoperative assessment, 88 degree postoperation and 87 degree at 18 months follow-up.
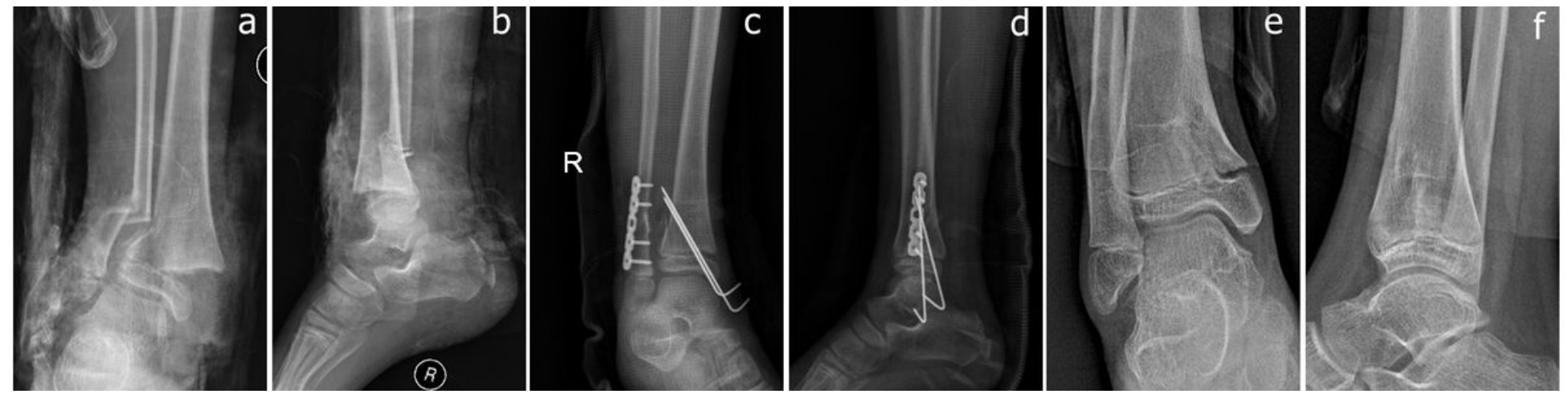

\section{Figure 4}

Boy, age 11, Case No.3, diagnosed the Dias-Tachdjian PEER physeal fracture of right distal tibial epiphysis and fibula, AOFAS-AH scoring 34 during preoperative assessment, 66 postoperation and 87 at 20 months follow-up. LDTA was 65 degree in the preoperative assessment, 87 degree postoperation and 105 degree at 20 months follow-up. $\mathrm{X}$ rays in the last follow up, shows bone bridge and varus of ankle joint. 